\title{
Pituitary adenylate cyclase-activating polypeptide mRNA expression in rat testis and epididymis during postnatal development and experimental cryptorchidism
}

\author{
CHUN-MEI LV*, DAN-LING CHENG* ${ }^{*}$, WEI ZHAO and HUI ZHU \\ Department of Physiology, Harbin Medical University, Harbin 150081, P.R. China
}

Received February 7, 2011; Accepted May 27, 2011

DOI: $10.3892 / \mathrm{mmr} .2011 .507$

\begin{abstract}
In the present study, the expression of pituitary adenylate cyclase-activating polypeptide (PACAP) mRNA was investigated in rat testis and epididymis during postnatal development and experimental cryptorchidism using reverse transcription-polymerase chain reaction (RT-PCR) and in situ hybridization. The results revealed that i) in the testis, PACAP mRNA expression was first detected on day 20 after birth and gradually increased from day 20 to reach a maximum level on day 60, mainly in the spermatocytes and round spermatids; ii) in the epididymis, PACAP mRNA was first detected on day 10 after birth and remained at higher levels from day 40; iii) the levels of PACAP mRNA in the testis and epididymis markedly decreased in a time-dependent manner from 1 week after cryptorchid surgery until they had almost disappeared at 4 weeks. These results suggested that the pattern of PACAP mRNA expression is developmentally dependent in both the testis and epididymis, and the expression of PACAP mRNA is regulated by the local temperature within the testis. In conclusion, the pattern of expression of PACAP in the testis and epididymis suggests a possible role of PACAP in spermatogenesis and sperm maturation.
\end{abstract}

\section{Introduction}

Pituitary adenylate cyclase-activating polypeptide (PACAP) is a member of the secretin/glucagon/vasoactive intestinal peptide (VIP) family of peptides. PACAP, originally isolated from ovine hypothalamic extract (1), exists in two active forms: PACAP38, with 38 amino acids and PACAP27, containing the N-terminal 27 amino acids of PACAP38.

Correspondence to: Dr Hui Zhu, Department of Physiology, Harbin Medical University, Harbin 150081, P.R. China

E-mail: dzhuhui@yahoo.com.cn

*Contributed equally

Key words: pituitary adenylate cyclase-activating polypeptide, testis, epididymis, cryptorchidism
PACAP has a widespread distribution in the central nervous system as well as in numerous peripheral tissues of mammals (2). Unexpectedly, the highest concentration of PACAP is found in the adult testis of rats; the total amount of PACAP in both testes exceeding that found in the entire brain (3). Recently, a study showed that PACAP was also highly represented in the testis of non-mammalian vertebrates, such as the crested newt Triturus carnifex and the wall lizard Podarcis sicula (4). Northern blotting and in situ hybridization analysis of rat testis demonstrated that PACAP mRNA is expressed in the developing germ cells in a stage-specific manner (5). Using immunohistochemistry, PACAP was observed in elongated spermatids and in the acrosomes of round spermatids in certain seminiferous tubules (6). These findings suggest that PACAP may function as a paracrine or autocrine regulatory factor in developing germ cells of testis during spermatogenesis. The epididymis provides an adequate environment for the final maturation of sperm. Therefore, the proper development and functional maintenance of the epididymis are essential for normal fertility. A previous study has reported that, in the rat epididymis, the intensity of immunoreactivity for PACAP was stronger in the caput and corpus regions of epididymidis (7) and that epididymal chloride secretion may be regulated by PACAP38 in a paracrine or autocrine fashion (8), suggesting that epididymal and sperm functions are subject to control by a local PACAP system in the rat epididymis. Together with the previous finding, PACAP may function in the male reproductive system.

Despite the abundance of PACAP in the adult testis and epididymis, to date little is known about the PACAP expression pattern in the testis and epididymis during the development of animals. Therefore, in the present study, PACAP mRNA expression was examined in rat testis and epididymis during postnatal development in order to investigate the functions of PACAP in the development and functional maintenance of the male reproductive system.

The testes of most mammals are located within a scrotum, the temperature of which is maintained $2-8^{\circ} \mathrm{C}$ lower than the temperature of the body cavity. The lower temperature is essential for normal testicular function. Cryptorchidism is the failure of the testis to descend from the abdomen into the scrotum. The rising temperature of the testis results in degenerative changes in the germinal epithelium and decreased 
fertility. However, the mechanisms mediating these degenerative changes are uncertain. Certain studies have shown that high temperatures induce an increased synthesis of several proteins and a decrease of many others (9). One previous study has shown that the germ cell loss associated with cryptorchidism occurs by apoptosis (10), which is evident primarily in pachytene spermatocytes within 2-4 days, as well as in the epithelial cells of the cauda epididymis.

However, the physiological functions of PACAP in the male reproductive system, and the relationship between PACAP expression and cryptorchidism remain uncertain. Thus, the other aim of the present study was to investigate the regionalization of the effect of cryptorchidism on PACAP mRNA expression in rat testis and epididymis in order to gain further insight into the possible role of PACAP in male reproduction.

\section{Materials and methods}

Animal treatments. All animal procedures were approved by the Institutional Animal Care and Use Committee of Harbin Medical University. Male rats (Wistar) were housed in a controlled environment (14L:10D) with standard laboratory chow and water ad libitum.

Postnatal development. The rats were sacrificed by decapitation at 10,20,30, 40 or 60 days after birth. The testis and epididymis from one side were collected, fixed in Bouin solution for $24 \mathrm{~h}$ and embedded in paraffin for in situ hybridization, whereas the contralateral testis and epididymis were frozen in liquid nitrogen for RNA extraction.

Unilateral cryptorchidism surgical procedure. Adult male Wistar rats (250-300 g) were divided into two groups. One group of animals was rendered unilaterally cryptorchid by surgery for 1,2, 3 and 4 weeks. The cryptorchid procedure was carried out by performing left inguinal canal closure through the inguinoscrotal approach (11). The other group of animals was sham operated as the controls. Surgical procedures were performed under sterile conditions. The rats were sacrificed by decapitation. The testes and epididymides were carefully collected and treated as above, respectively.

Reverse transcription-polymerase chain reaction (RT-PCR). The testis and epididymis were removed, weighed, quick frozen in liquid nitrogen and homogenized in TRIzol solution (Gibco Brl., Gaithersburg, MD, USA). Total RNA was extracted according to the manufacturer's instructions and $2 \mu \mathrm{g}$ of the total RNA was reverse-transcribed into cDNA using an oligo(dT) primer. PCR amplification was performed by using the cDNA template with the specific primer pairs (5'-ATCAGACCAG AAGAGGC-3' and 5'-GCTATTCGGCGTCCTTTGTT-3'). Standardization of basal levels between samples was made possible by using GAPDH as a housekeeping gene. The amplified products were detected by electrophoresis in a $1.5 \%$ agarose gel.

In situ hybridization. Tissues were fixed in Bouin's solution at room temperature overnight. The fixed tissues were dehydrated, embedded in paraffin and sectioned at a thickness of $5 \mu \mathrm{m}$. Paraffin sections were transferred onto slides and dried overnight at $37^{\circ} \mathrm{C}$. Sections were deparaffinized and rehydrated through an alcohol series. Sections were incubated in $0.3 \% \mathrm{H}_{2} \mathrm{O}_{2}$ for $10 \mathrm{~min}$, rinsed thoroughly with PBS and then permeabilized with proteinase $\mathrm{K}$ for $5 \mathrm{~min}$ at room temperature. After washing for $3 \times 5$ min with PBS, the sections were prehybridized for $3 \mathrm{~h}$ at $37^{\circ} \mathrm{C}$ followed by hybridization with digoxigenin-labeled PACAP cRNA probe overnight at $37^{\circ} \mathrm{C}$. After hybridization, the sections were washed with $2 \mathrm{X}$ SSC for $10 \mathrm{~min}, 1 \mathrm{X} \mathrm{SSC}$ for $15 \mathrm{~min}$ and $0.2 \mathrm{X} \mathrm{SSC}$ for $10 \mathrm{~min}$ at $37^{\circ} \mathrm{C}$. The sections were then treated with phosphatase-labeled antidigoxigenin antibody at $37^{\circ} \mathrm{C}$ for $1 \mathrm{~h}$ and washed with PBS. The reaction was visualized using diaminobenzidine (DAB; Dako Corp., Carpinteria, CA, USA) as a substrate. Slides were counterstained with hematoxylin. After dehydration by passing the slides through a series of graded ethanol solutions, they were mounted. For each test, control experiments were performed using prehybridization instead of the probe.

\section{Results}

Expression of PACAP $m R N A$ in rat testis during postnatal development. PACAP mRNA expression was examined in rat testis during postnatal development by RT-PCR and in situ hybridization. RT-PCR showed that basal PACAP mRNA was detected on day 20 after birth. With the development of the testis, the level of PACAP mRNA expression gradually increased and reached a maximum in the young adult by day 60 (Fig. 1A). Distribution of PACAP mRNA in rat testis was detected by in situ hybridization. PACAP mRNA was not detected on day 10 (Fig. 1B-a). A weak signal of PACAP mRNA was seen in the seminiferous tubules on day 20 (Fig. 1B-b). As cellular content becomes more complex at later time-points, the PACAP signal is associated with cells located more towards the center of developing tubules. PACAP expression gradually increased from day 30 (Fig. 1B-c), and significantly increased on day 40 after birth (Fig. 1B-d). Distribution of PACAP mRNA is mainly localized in the spermatocytes and round spermatoa of the testis in the adult rat (Fig. 1B-e). No positive signals were observed in the testis when prehybridization was used to replace the PACAP cRNA probe (Fig. 1B-f).

Expression of PACAP mRNA in rat epididymis during postnatal development. RT-PCR showed that in the caput and cauda epididymis, a very low level of PACAP mRNA was detected on day 10 after birth. A basal expression was seen in the caput and cauda epididymis on days 20 and 30. There was a high level of PACAP mRNA on day 40 which remained at higher levels in all portions of the epididymis on day 60 (Fig. 2A). Distribution of PACAP mRNA in rat epididymis during postnatal development was detected by in situ hybridization. PACAP mRNA expression was first detected in the epididymal epithelium of the caput and cauda epididymis at low levels on day 10 after birth and had steadily increased by days 20 and 30 (data not shown). High expression levels of PACAP mRNA were observed on day 40 after birth and these levels remained high until day 60 (Fig. 2B). No positive signals were observed in the epididymis when prehybridization was used to replace the PACAP cRNA probe (data not shown). 


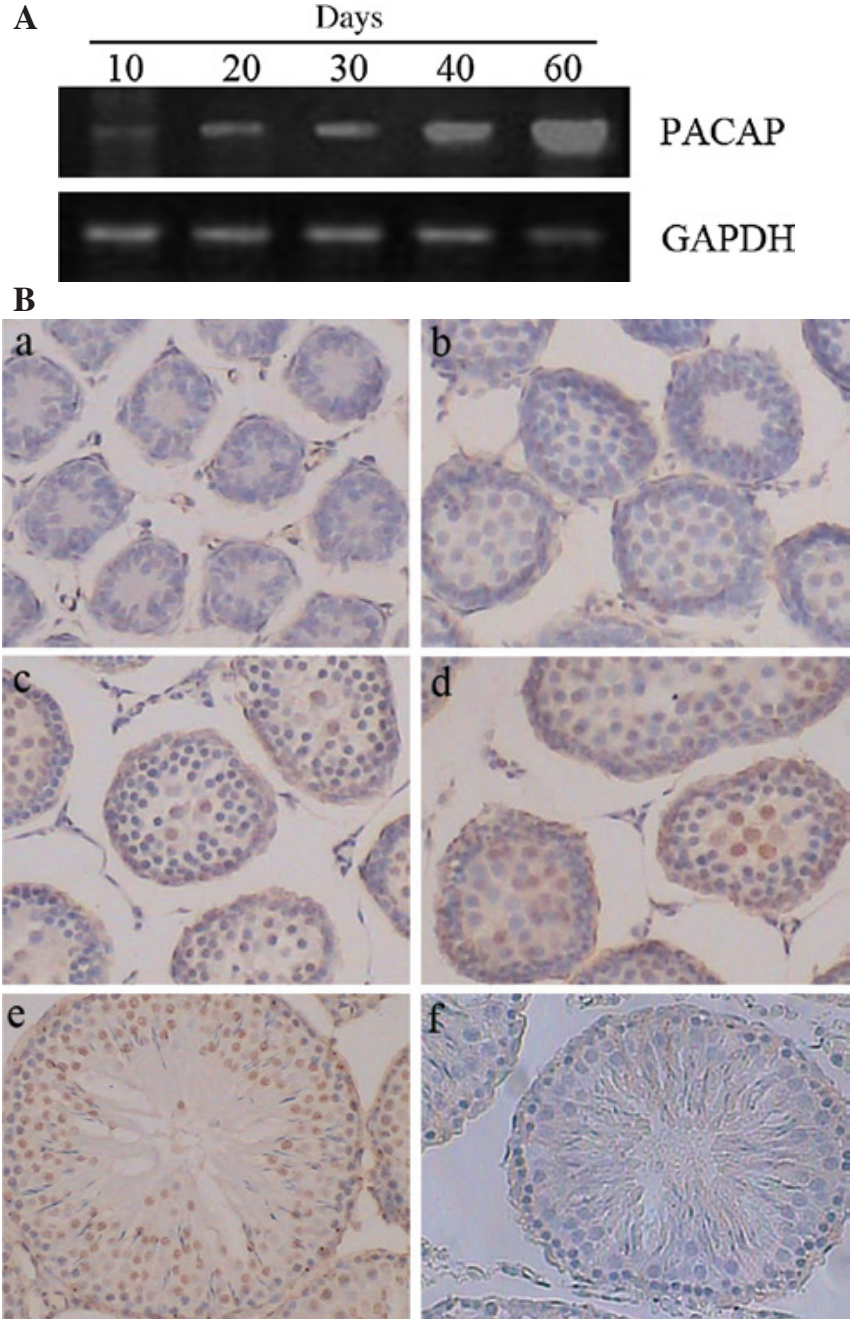

Figure 1. Expression of PACAP mRNA in rat testis during postnatal development. (A) PACAP mRNA expression in rat testis by RT-PCR. (B) In situ hybridization of PACAP mRNA in rat testis on (a) day 10, (b) day 20, (c) day 30, (d) day 40 and (e) day 60; (f) negative control. Magnification, x200.

Effects of artificial cryptorchidism on testicular and epididymal PACAP mRNA expression. To examine the effects of heat stress on PACAP mRNA expression in the testis and epididymis in vivo, experimental cryptorchidism was employed. RT-PCR revealed that the level of PACAP expression decreased abruptly in the cryptorchid testis and epididymis at 1 week after surgery. It is noteworthy that PACAP mRNA levels continued to decline following the induction of cryptorchidism and were almost extinguished at 4 weeks after surgery in the testis and in the caput and cauda epididymis (Fig. 3A). Distribution of the PACAP mRNA was examined by in situ hybridization at various times after the induction of artificial cryptorchidism in the testis and epididymis. In the control testis, PACAP mRNA was present in the spermatocytes and round spermatids, but not in spermatozoa (Fig. 3B-a), whereas in the control epididymis it was found mainly in the epididymal epithelia (Fig. 3B-d and g). The number of positive cells decreased after the induction of cryptorchidism in the testis and epididymis compared to the controls, and there were trace levels on week 2 after surgery both in the testis (Fig. 3B-b) and epididymis (Fig. 3B-e and h). After 4 weeks of cryptorchidism, PACAP mRNA expression had decreased
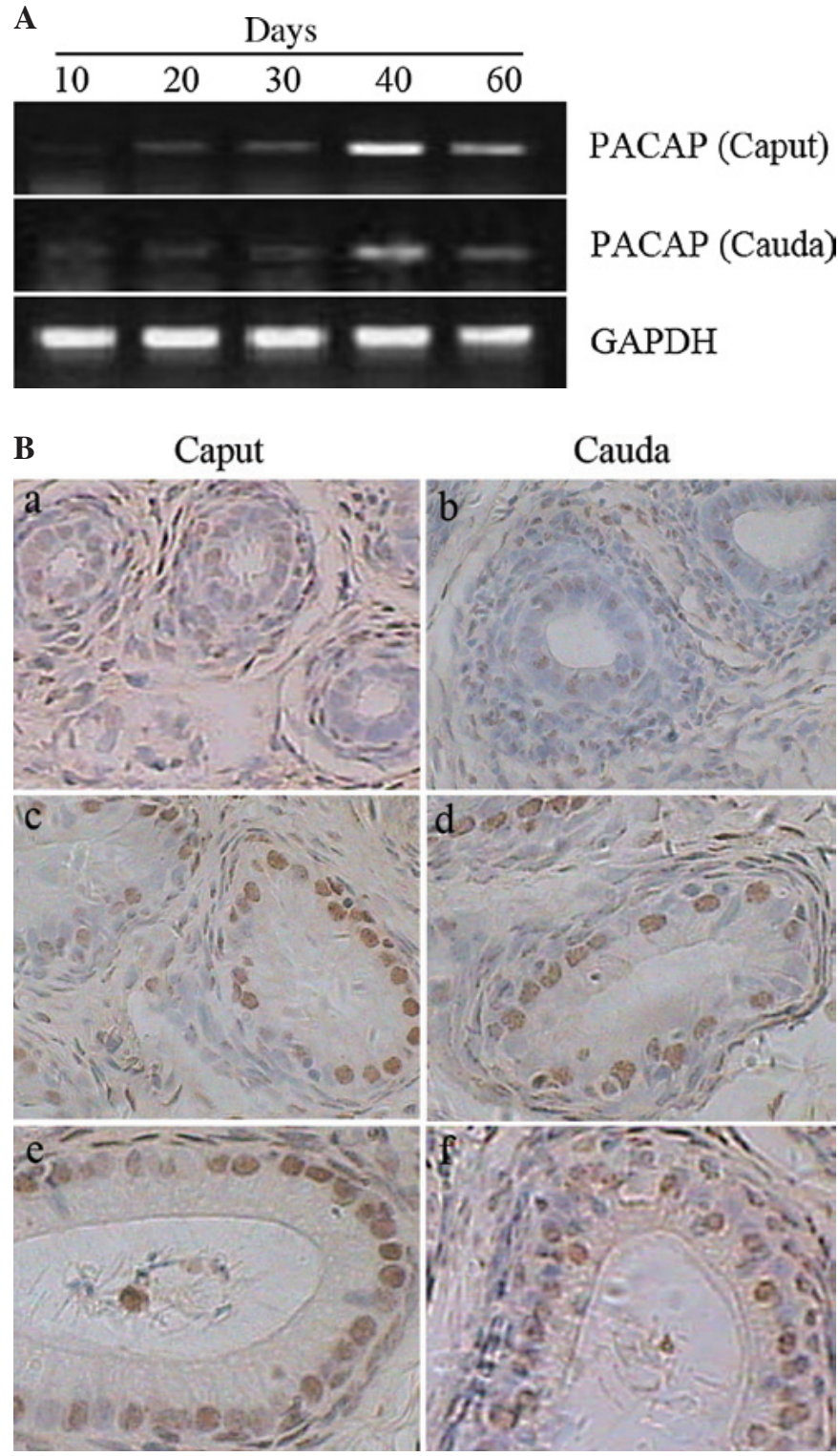

Figure 2. Expression of PACAP mRNA in rat epididymis during postnatal development. (A) PACAP mRNA expression in the caput and cauda of rat epididymis by RT-PCR. (B) In situ hybridization of PACAP mRNA in the caput and cauda of rat epididymis on (a and b) day 10, (c and d) day 40 and (e and f) day 60. Magnification, x200.

to a barely detectable level in the cryptorchid side (Fig. 3B-c, $\mathrm{f}$ and i). On histological sections of the cryptorchid testis, no spermatozoa and spermatids, fewer spermatocytes and mostly spermatogonial cells could be observed, suggesting an arrest of spermatogenesis at the meiotic stages. By contrast, spermatogenesis in the contralateral testis was normal.

\section{Discussion}

In the present study, the expression of PACAP mRNA was investigated in rat testis and epididymis during postnatal development and experimental cryptorchidism using RT-PCR and in situ hybridization.

The results demonstrated that before sexual maturity, on day 10 after birth, the seminiferous tubules were very small and epithelial cells had not developed in the tubules. PACAP mRNA was very low. As development progressed, the level of 
A

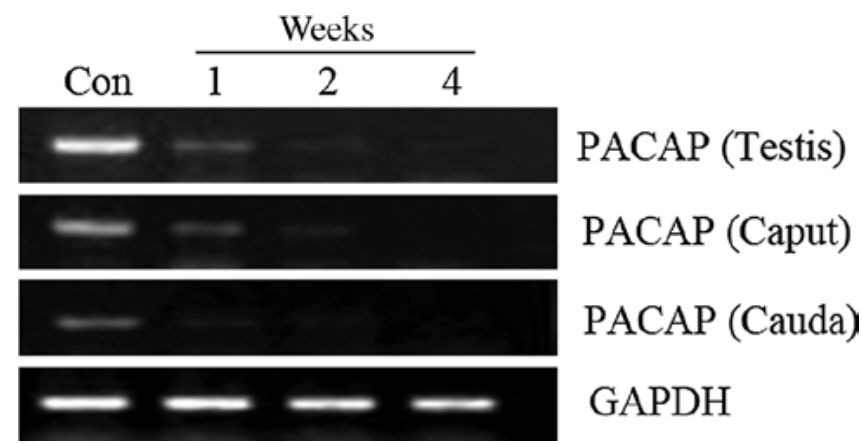

B

Control

2 weeks

4 weeks
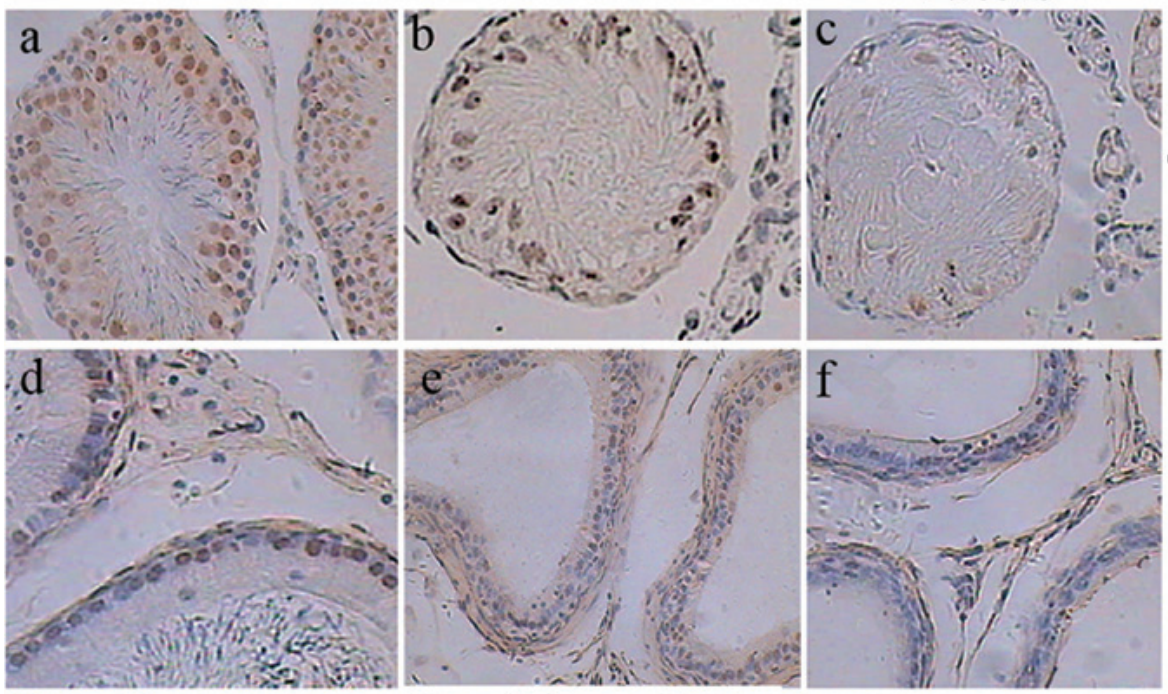

Caput
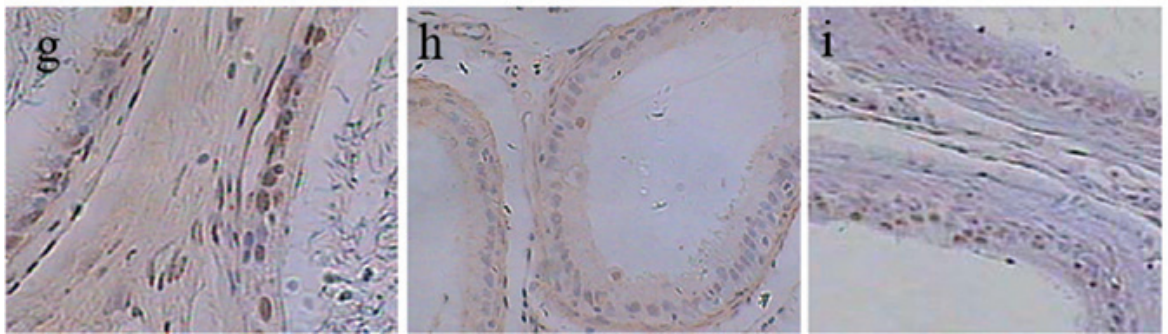

Testis

Cauda

Figure 3. Expression of PACAP mRNA in the testis and epididymis of cryptorchid rat. (A) PACAP mRNA expression in the testis and epididymis of cryptorchid rat by RT-PCR. Con, control; weeks (1,2 and 4), weeks after surgery. (B) Localization of the PACAP mRNA in the testis and epididymis of cryptorchid rat by in situ hybridization. 2 weeks and 4 weeks, weeks after surgery. Magnification. x200.

PACAP mRNA in rat testis gradually increased. Several studies have shown that PACAP stimulates the proliferation and differentiation of various types of cells. PACAP injections in P8 rats cause an increase in the thickness of the cell layer. PACAP also promotes cell survival and neurite outgrowth in cultured immature granule cells. In the septum and hypothalamus, the content of PACAP increases gradually from birth to postnatal day 60 (12), suggesting that PACAP may control the proliferation or differentiation of neuroblasts during neural tube development. Similarly, PACAP may contribute to the proliferation or differentiation of the germ cell during testicular development. In adult rat testis, the expression of PACAP mRNA is mainly localized in spermatocytes and round spermatids, but not in spermatozoa. These data are consistent with the finding of Koh et al (13) - that the role of PACAP in testicular development and function may be age-dependent. It has been reported that the cAMP-responsive element modulator (CREM) protein is abundant in adult rat testis and is highly expressed in round spermatids, predominantly at stages VII and VIII of spermatogenesis, but not in spermatozoa (14), similar to the expression of testicular PACAP. CREM activation may be directly regulated by the CAMP pathway in germ cells and appears to regulate the differentiation processes of the haploid germ cells (14). It is well known that the junctions form the most effective part of the blood-testis barrier that restricts the free passage of a number of compounds. Since it may be difficult for many circulating factors to enter the seminiferous tubules, the testis must produce many of its own regulatory substances. PACAP mRNA was detectable in the testis, primarily in developing germ cells. Therefore the site- and stage-specific expression of PACAP and CREM in the spermatids support the view that testicular PACAP synergistically promotes the activation of CREM, thereby regulating spermatogenesis.

In this study, PACAP mRNA expression levels in the caput and cauda epididymis were also examined during development. The development of the rat epididymal epithe- 
lium during the postnatal period has been divided into three stages; the undifferentiated stage, the differentiation stage and the expansion stage (15). PACAP mRNA expression was first detected on day 10 after birth in the epididymal epithelia of the caput and the cauda epididymis. At this time, the epididymal epithelium is essentially undifferentiated. When the epididymal epithelium begins to differentiate, PACAP mRNA concentration increases steadily during maturation. The highest expression of PACAP mRNA was observed on day 40 after birth and at that time dramatic morphological changes occurred in the epididymal epithelium (15). In young adults, epididymal epithelia were fully differentiated and PACAP mRNA exhibited a region-specific expression; by RT-PCR analysis, it was shown that PACAP mRNA level was the highest in the caput, followed by the cauda of rat epididymis. The results indicate that PACAP mRNA expression during the postnatal period is closely associated with the differentiation of the epididymal epithelium. This regional specificity of PACAP mRNA expression should be related to corresponding functions in each part of the epididymis. The acquisition of the sperm motility and zona pellucida binding capacity mainly occurs in the caput and corpus epididymis, whereas the cauda epididymis specializes in sperm storage (16). The three regions of the epididymis are different in their epithelial cell morphology and specific pattern gene expression (17). However, the significance of the region-specific spatial and temporal expression of PACAP in rat epididymis remains unknown.

Mammalian spermatogenesis typically occurs at a temperature a few degrees lower than core body temperature, which is attained by the descent of the testis into the scrotum. Cryptorchidism is the failure of the testes to descend from the abdomen to the scrotum. The present study, using surgically-induced unilateral cryptorchidism, demonstrates that the level of PACAP mRNA expression dropped rapidly from 1 week after cryptorchidism compared to the control, and had almost disappeared 4 weeks after cryptorchidism. The earliest cellular changes reported in experimentally cryptorchid testes were in pachytene spermatocytes and early spermatids, and experiments report that PACAP38 mRNA is only expressed in this phase of spermatogenesis. It has been reported that the germ cell loss associated with cryptorchidism occurs by apoptosis, and that temperature is one of the factors that trigger apoptosis in the epididymidis (18). Several lines of evidence suggest that PACAP functions in protecting cells from apoptosis by different mechanisms. Certain studies have reported that PACAP acts on PACAP type I receptor (PAC1-R) to promote cerebellar neuron survival through the cAMP/PKA signaling pathway (19), and a study by Li et al found that cytosolic endogenous PACAP in the spermatids was co-immunoprecipitated with the cytosolic PAC1-R and markedly stimulated the expression and activation of MAPK (20). Thus, cAMP/PKA signaling seems to be required to protect cells from apoptotic events, ensuring proper cell development. However, in chick sympathetic neuroblasts, PACAP rescues cells from death, but does not act through the usual pathways. Instead, PACAP appears to operate through an unidentified receptor to decrease the concentration of a death protein by stimulating the destruction of the protein. Another report has shown that PACAP prevents neuronal cell death after ischemia via a signaling mechanism involving IL-6 (21). Based on the results of this and previous studies, it is proposed that the decrease in PACAP expression is related to the apoptosis of the germ cells and epididymal epithelia in experimental cryptorchidism.

In summary, this study demonstrates the expression of PACAP mRNA in rat testis and epididymis during postnatal development and experimental cryptorchidism. Taken together, the data in this study suggest that PACAP may play crucial roles in testicular and eipididymal development, and that it may be involved in spermatogenesis and sperm maturation in male reproduction.

\section{Acknowledgements}

This study was supported by the Heilongjiang Province Educational Commission of China (No. 1055 G024).

\section{References}

1. Miyata A, Arimura A, Dahl RR, et al: Isolation of a novel 38 residue-hypothalamic polypeptide which stimulates adenylate cyclase in pituitary cells. Biochem Biophys Res Commun 164: 567-574, 1989.

2. Vaudry D, Falluel-Morel A, Bourgault S, Basille M and Burel D: Pituitary adenylate cyclase-activating polypeptide and its receptors: 20 years after the discovery. Pharmacol Rev 61: 283-357, 2009.

3. Arimura A, Somogyvári-Vigh A, Miyata A, Mizuno K, Coy DH and Kitada C: Tissue distribution of PACAP as determined by RIA: Highly abundant in the rat brain and testes. Endocrinology 129: 2787-2789, 1991.

4. Agnese M, Valiante S, Angelini F, Laforgia V, Andreuccetti P and Prisco M: Pituitary adenylate cyclase-activating polypeptide and its receptor PAC1 in the testis of Triturus carnifex and Podarcis sicula. Gen Comp Endocrinol 168: 256-261, 2010.

5. Kononen J, Paavola M, Penttilä TL, Parvinen $M$ and Pelto-Huikko M: Stage specific expression of pituitary adenylate cyclase-activating polypeptide (PACAP) mRNA in the rat seminiferous tubules. Endocrinology 135: 2291-2294, 1994.

6. Lissbrant E, Collin O and Bergh A: Pituitary adenylate cyclaseactivating polypeptide (PACAP): effects on blood flow in the testis and caput epididymidis of the rat. J Androl 20: 366-374, 1999.

7. Leung PS, Wong TP, Wong PY and Chan HC: Localization and distribution of pituitary adenylate cyclase-activating polypeptide in the rat epididymis. Cell Biol Int 22: 193-198, 1998.

8. Zhou WL, Leung PS, Wong TP, Dun NJ, Wong PYD and Chan HC: Local regulation of epididymal anion secretion by pituitary adenylate cyclase-activating polypeptide. J Endocrinol 154: 389-395, 1997.

9. Cataldo L, Mastrangelo MA and Kleene KC: Differential effects of heat shock on translation of normal mRNAs in primary spermatocytes, elongated spermatids, and Sertoli cells in seminiferous tubule culture. Exp Cell Res 231: 206-213, 1997.

10. Kirchhoff C: Gene expression in the epididymis. Int Rev Cytol 188: 133-202, 1999.

11. Dundar M, Kocak I and Culhaci N: A new experimental model for cryptorchidism: inguinoscrotal approach. Urol Res 29: 178-181, 2001.

12. Masuo Y, Tokito F, Matsumoto Y, Shimamoto N and Fujino M: Ontogeny of pituitary adenylate cyclase-activating polypeptide (PACAP) and its binding sites in the rat brain. Neurosci Lett 170: 43-46, 1994.

13. Koh PO, Noh HS, Kim YS, et al: Cellular localization of pituitary adenylate cyclase-activating polypeptide in the rat testis. Mol Cells 15: 271-276, 2003.

14. Delmas V, Hoorn FVD, Mellstrom B, Jegou B and Sassone-Corsi P: Induction of CREM activator proteins in spermatids: downstream targets and implications for haploid germ cell differentiation. Mol Endocrinol 7: 1502-1514, 1993.

15. Robaire B and Hermo L: Efferent ducts, epididymis and vas deferens: structure, functions and their regulation. In: The Physiology of Reproduction. Knobil E and Neill J (eds). Raven Press, New York, pp999-1079, 1988. 
16. Moore HD: Contribution of epididymal factors to sperm maturation and storage. Andrologia 30: 233-239, 1998.

17. Doiron K, Légaré C, Saez F and Sullivan R: Effects of vasectomy on gene expression in the epididymis of cynomolgus monkey. Biol Reprod 68: 781-788, 2003.

18. Marco J, Pedro E and Rosa C: Abdominal temperature induces region-specific p53-independent apoptosis in the cauda epididymidis of the mouse. Biol Reprod 67: 1189-1196, 2002.

19. Mei YA, Vaudry D, Basille M, Castel H, Fournier A, Vaudry H and Gonzalez BJ: PACAP inhibits delayed rectifier potassium current via a cAMP/PKA transduction pathway: evidence for the involvement of I k in the anti-apoptotic action of PACAP. Eur J Neurosci 19: 1446-1458, 2004
20. Li M, Funahashi H, Mbikay M, Shioda S and Arimura A: Pituitary adenylate cyclase activating polypeptide-mediated intracrine signaling in the testicular germ cells. Endocrine 23: 59-75, 2004.

21. Ohtaki H, Nakamachi T, Dohi K, et al: Pituitary adenylate cyclase-activating polypeptide (PACAP) decreases ischemic neuronal cell death in association with IL-6. Proc Natl Acad Sci USA 103: 7488-7493, 2006. 